Further Section

Neuroepidemiology 1983;2:277

\title{
Author Index Vol. 2, 1983
}

Alexander, V. 179 Angelini, C. 236 Armani, M. 236

Bahemuka, M. 196 Beghi, E. $\quad$ 156,270 Bharucha, N.E. 148 Bolis,C.L. 121 Bresolin, N. 236

Capildeo.R. 135 Chandra, V. 148 Cheng, X.-M. 121 Clark, V.A. 56 Clifford Rose, F. 135 Cooper, K.D. 70

Dastur.D.K. 93 Detels, R. 45, 56 Dudley, J.P. 45, 56

Escobedo-Rios, F. 16

Factor, P.R. 70 Feiner, C. 70 Fratiglioni, L. 101

Gajdusek, D.C. 24, 35 Garcia-Pedroza, F. 16 Garcia-Ramos, G. 16 Gonzalez-Cortes, A. 16 
Goudsmit, J. 24, 35 Greenland, S. 5

Haberman, S. 135 Halperin,W. 179 Hauser, W.A. 70 Henderson, B.E. 164

Ikeda,M. 62

Karavodin, L.M. 45 Kurland, L.T. 89,156,206

270 Kuroiwa, Y. 62

Layton,D. 206 Leffingwell, S.S. 179 Li,S.-Z. 121 Ludwig, H.R. 179

Malmgren, R.M. 56 Massey.E.W. 101,

196 Molina, R. 219 Mulder, D.W. 270

Poser, CM. 266 Preston-Martin, S. 164

Ragozzino, M.W. 89 Rajput, A.H. 89 
Ramirez-Lassepas, M.

219 Rubio-Donnadieu, F. 16

Salguero, L.F. 219 Sasanelli, F. 156 Scarlett Kranz, J.M. 206 Schoenberg, B.S. 1, 101, 117, 121, 148, 196,257 Schoenberg, D.G. 101 Schuman, L.M. 206 Shibasaki, H. 62 Shimada, Y. 243 Shulman,K. t 70 SinghaLB.S. 93 Strassburg, M.A. 5 Sullivan, C.B. 45

Tabaddor.K 70 Tanaka,H. 243 Toyokura, Y. 243

Visscher, B.R. 56

Waals, F.W. van der 24,

35 Wang,C.-C. 121 Waxweiler, R. 179

Yase,Y. 243 Yu,M.C. 164 